\title{
Concentric "Microwaves" of Henle's Fiber Layer: Associated with Horizontal Folding
}

\section{J. Reimer Wolter}

The Departments of Opthalmology and Pathology of the University of Michigan Medical Center, Ann Arbor, Michigan 48109, USA

\begin{abstract}
Concentric "microwaves" in Heale's fiber layer are found in a case of extensive horizontal folding in the fundus due to tumor pressure on the globe from behind. In addition to horizontal folding of inner choroid and inner retina between disk and fovea, somewhat irregular vertical folds are found next to the fovea in the inner retinal layers. The concentric waves of Henle's fiber layer are a new observation and they are suspected to be a protective adjustment of the central retina to minimize distortion of the foveola.

Zusammenfassung. Konzentrische Mikrowellen in Henle's Faserschicht werden in einem Fall von horizontaler Faeltelung im Augenhintergrund bei Tumordruck von hinten dargestellt. Zusätzlich zu horizontalen Falten der Aderhaut und Netzhaut zwischen Sehnerv und Fovea werden leicht unregelmäßige vertikale Falten der inneren Netzhaut im Foveabereich gefunden. Die konzentrischen Mikrowellen in Henle's Faserschicht sind eine neue Beobachtung und dieselben könnten eine mechanische Anpassung der zentralen Netzhaut zur Vermeidung von Verzerrung der Fovea darstellen.
\end{abstract}

Parallel folds or striae may occur in the posterior fundus as a result of longstanding pressure on the posterior aspect of the eye in orbital tumors or in Graves disease, for example $[3-6,9,12]$. Similar folding may be seen as a result of ocular hypotony [1]. Folds of this type are usually associated with a change of the refractive values towards hyperopia indicating shortening of the globe and most commonly they run in a horizontal direction and are most developed in the zone between disk and fovea. However, in rare cases the folds have been seen to run in vertical, oblique or even concentric directions $[2,8]$. The folds typically persist after the pressure on the posterior sclera has been relieved

Offprints requests to: J. Reimer Wolter, M.D., Department of Ophthalmology, University of Michigan Medical Center, Ann Arbor, Michigan 48109, USA 
- by removal of an orbital tumor, for example. However, transient folds have been reported in acute situations [7].

A number of good clinical, angiographic and histological $[10,11,13]$ studies have resulted in satisfactory understanding of the nature of the horizontal folds. It is important to emphasize that these folds may involve the retina, but the change in the ophthalmoscopic view is mainly caused by folding of the nonelastic membrane of Bruch with the pigment epithelium firmly attached to it. The folding of the pigment epithelium causes the clinically impressive picture of parallel alternating bright and dark lines. The retinal folds are much more difficult to see ophthalmoscopically than those of the choroid. The sclera simply adjusts by shrinkage and diffuse thickening.

Horizontal folds, surprisingly, do not interfere with central vision much in most cases even if the foveal region is clearly involved. Furthermore, one sometimes sees patients where the folding seems to avoid the foveal region (comp. Fig. 3-4, page 103 in Gass [2]). Thus, clinical observation seems to indicate that the foveola may be protected somehow and in some cases from involvement in this folding process.

The present paper aims to demonstrate the surprising changes found in Henle's fiber layer with the use of flat sectioning and silver staining in a case of clinically observed and histologically proven horizontal folding of Bruch's

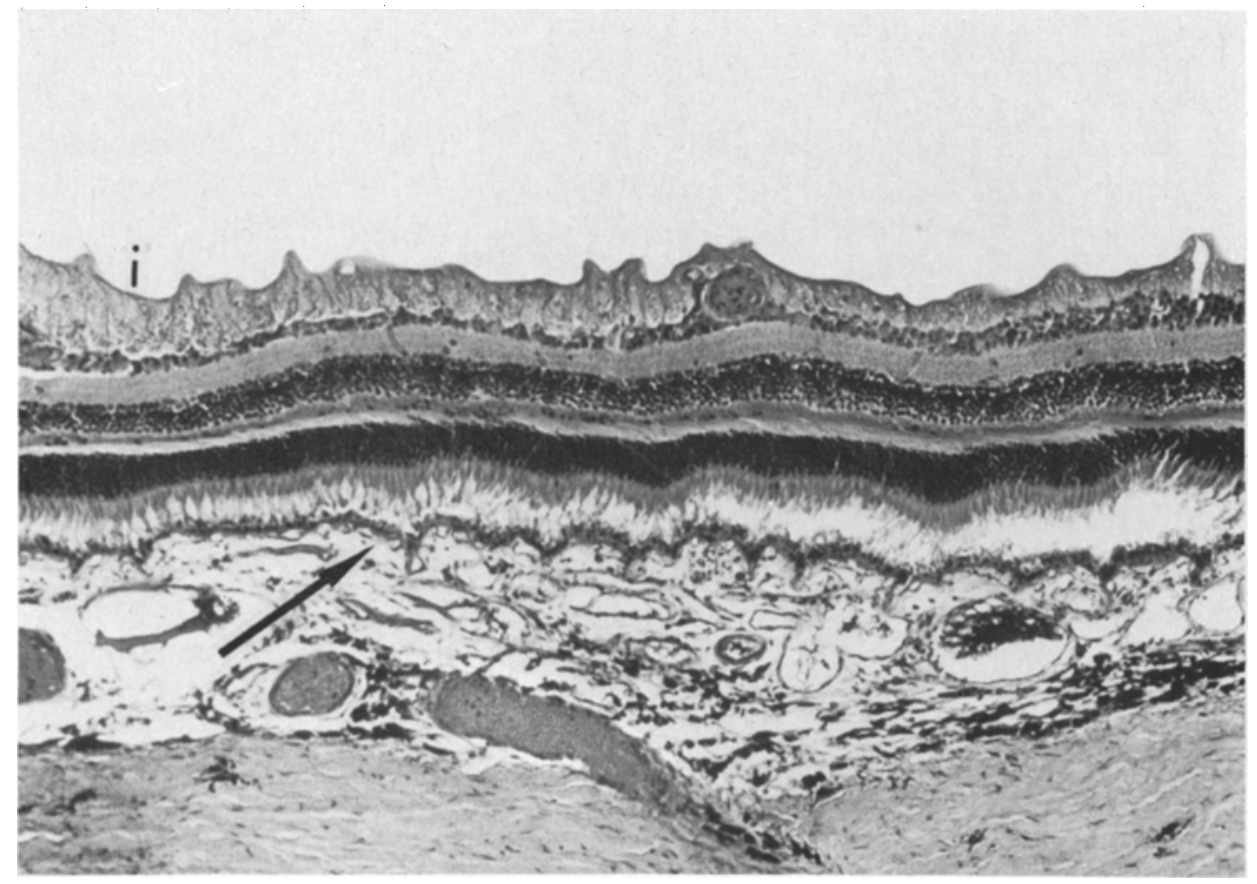

Fig. 1. (Wolter) Cross section of retina and choroid in a vertical plane shows distinct and irregular folding of the inner limiting membrane of the retina $(i)$ and the much more regular folding of Bruch's membrane with the pigment epithelium (arrow). - Paraffin section, $\mathrm{H}$ and $\mathrm{E}$ stain, photomicrograph $\times 150$ 
membrane and retina due to tumor pressure on the eye. These changes, possibly, represent a protective adjustment of the star-shaped structure of Henle's fiber layer that could very well prevent foveolar distortion.

\section{Case Report}

This again concerns the previously reported [10] 20 year-old female with a two year history of orbital extensions of an osteoblastic sarcoma. In spite of repeated surgical removal and radiation treatment severe proptosis developed. Horizontal choroidal folds formed and covered a fundus area of about five by eight disk diameters. The folds were highest between disk and fovea. Ophthalmoscopically the fovea was involved with the shallow temporal end of the horizontal folds. Vision

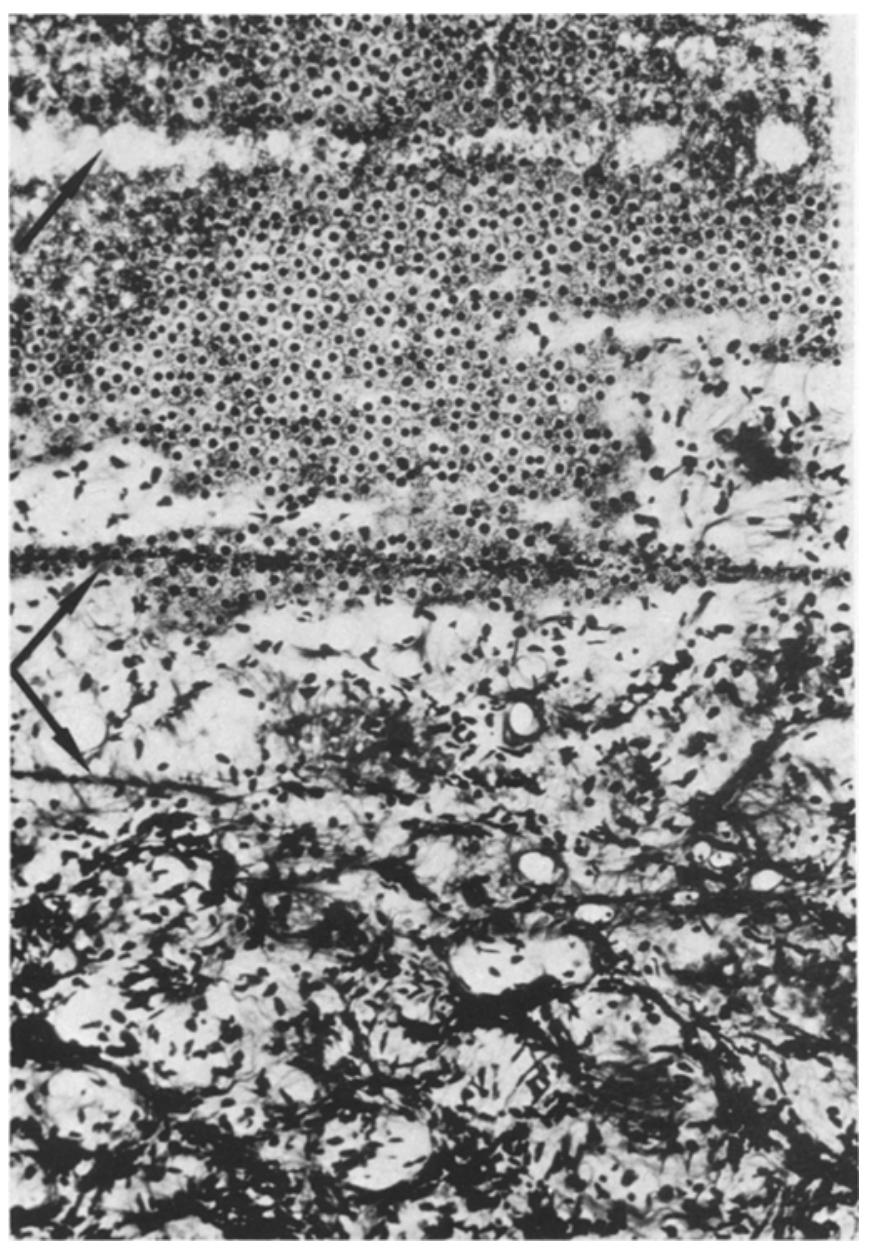

Fig. 2. (Wolter) In a transitional flat section of the foveal zone the pigment epithelium is seen on top and the choroid on the bottom of the photograph. Three horizontal folds are seen to involve these layers - and they look like ridges (arrow). The knife has cut the top off the upper ridge. Frozen section, Hortega stain, photomicrograph $\times 300$ 


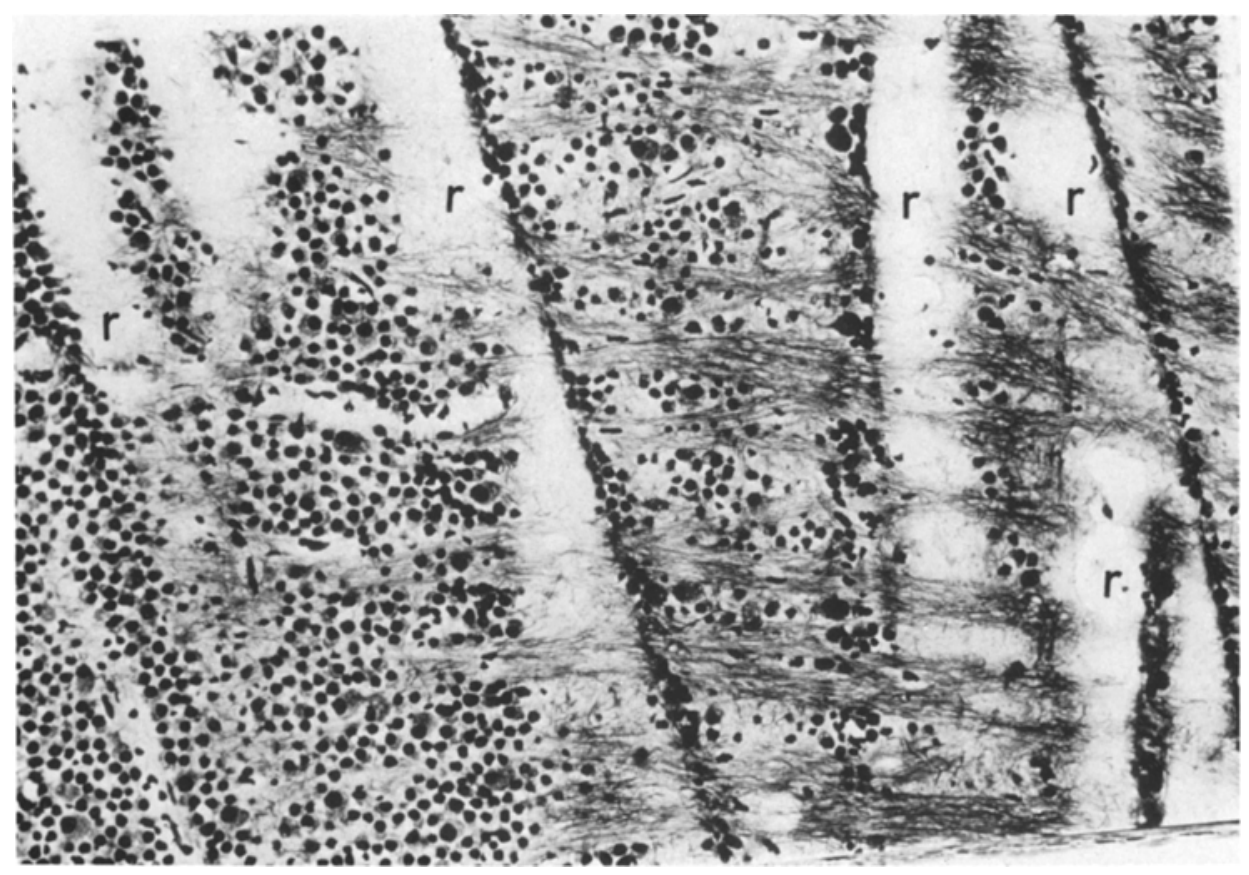

Fig. 3. (Wolter) Transitional flat section slightly superior and nasal to fovea going from nerve fiber layer (right) to ganglion cell layer (left) - exhibiting bundles of nerves in the nerve fiber layer. The light linear defects in the photograph represent vertical folds or ridges $(r)$ of a somewhat irregular arrangement. - Frozen section, Hortega stain, photomicrograph $\times 210$

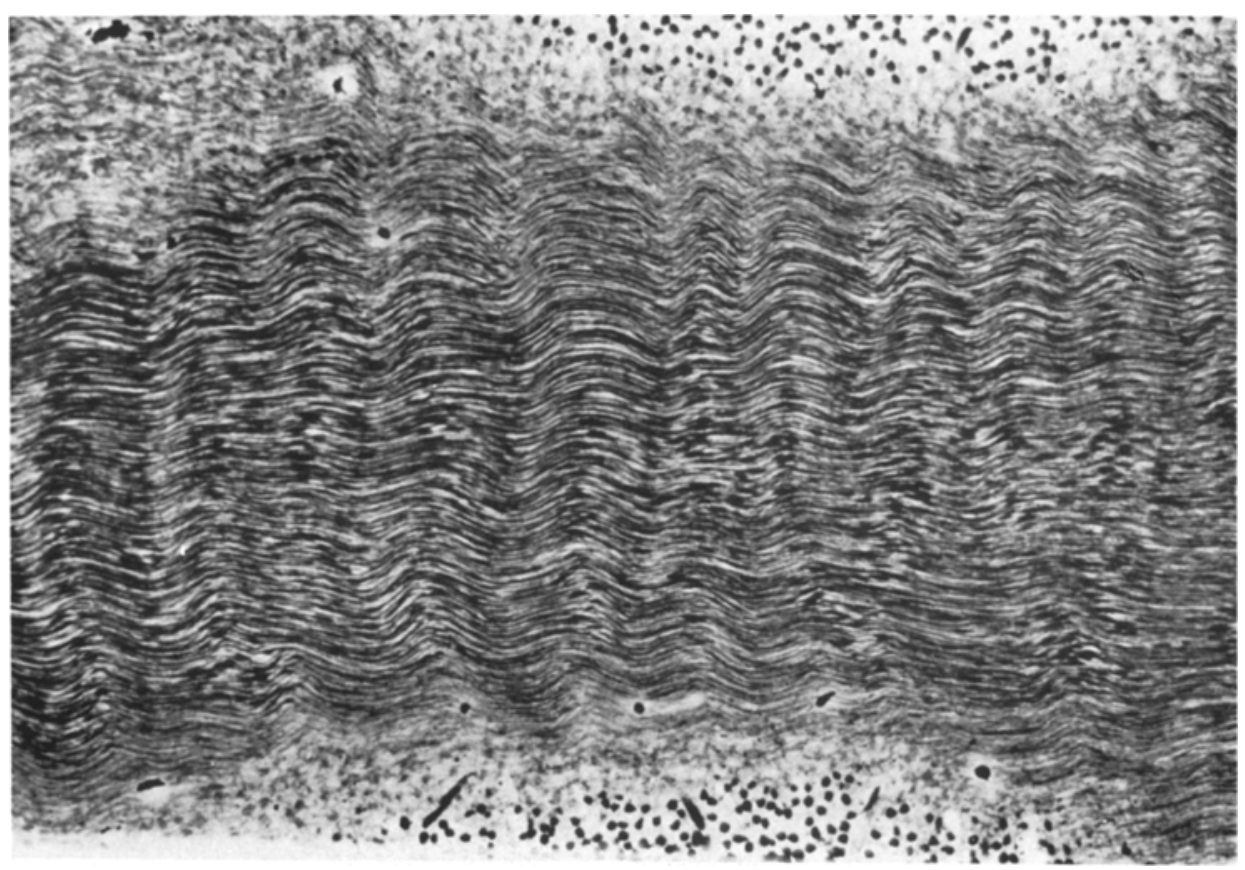

Fig. 4. (Wolter) A part of the temporal aspect of Henle's fiber layer exhibiting regular "microwaves" with ridges and valleys in a vertical direction. All fibers are well preserved. - Flat frozen section, Hortega stain, photomicrograph $\times 300$ 


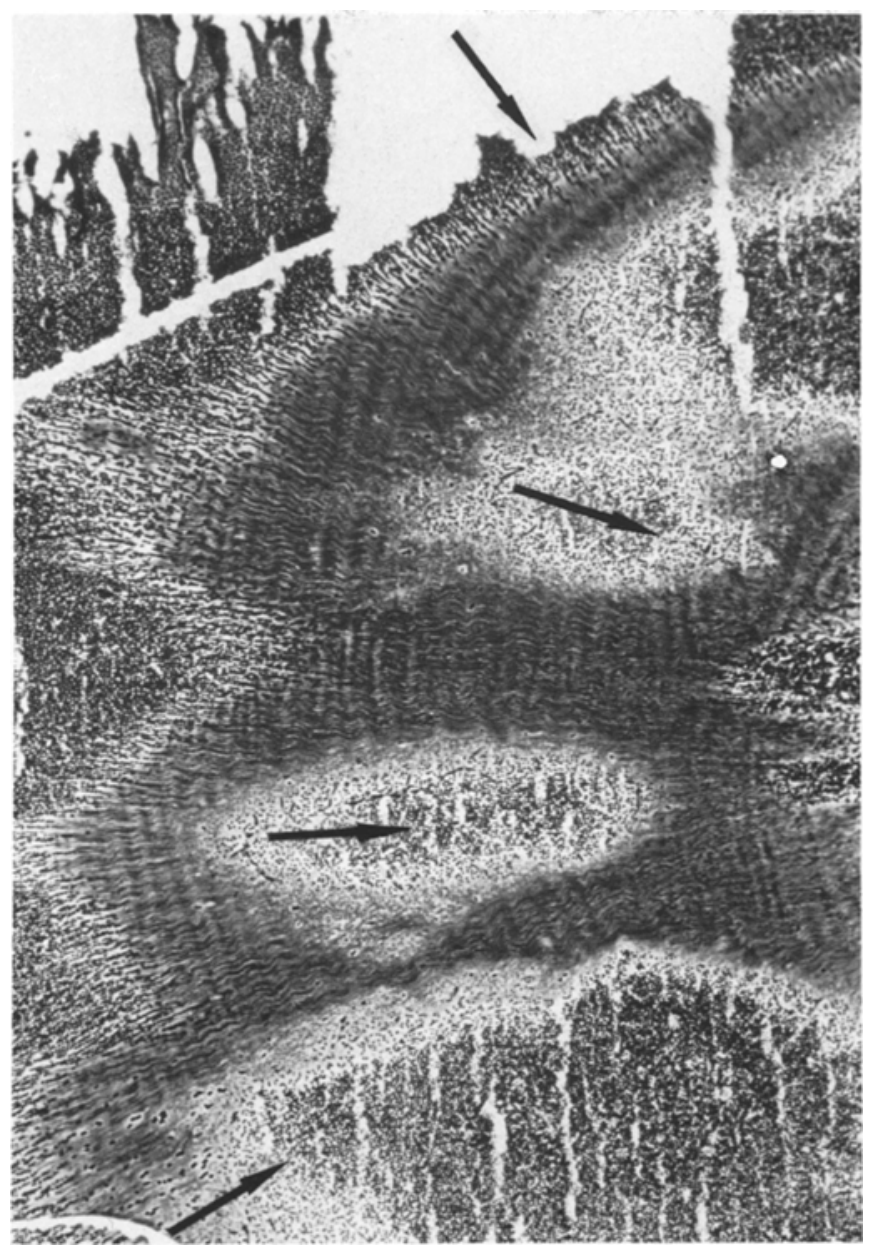

Fig. 5. (Wolter) Lower power view of flat section showing the ridges of the orderly folds in Henle's fiber layer to curve (arrow) around the foveola - located slightly right to the left border of the photograph. The freezing of the section was not totally level and, thus, the knife has not followed the level of Henle's fibers exactly. - Frozen section, Hortega stain, photomicrograph $\times 75$

was reduced to $6 / 15$ with a hyperopic correction, but there was some optic nerve atrophy and it was found that the optic nerve was directly involved with the orbital tumor extensions.

The eye was removed during exenteration of the orbit and Formalin was injected immediately to preserve the clinically observed horizontal folding. The eye was opened in a vertical plane for sectioning in the same direction. Histologically, folding of the inner retinal layers and Bruch's membrane was observed and recorded in detail [11]. Figure lgives a representation of the findings. It can be emphasized that in the region between disk and fovea the choroidal folds and the folds of the inner retinal layers, both ran in a horizontal direction.

The foveal region of the retina was isolated after completed fixation and frozen flat sections were stained with the silver carbonate technique of Del Ria Hortega. At that time the sections were considered normal except for folding of the inner limiting membrane and Bruch's membrane - which already had been recognized in the Paraffin cross sections. However, a recent review of the slides revealed a noteworthy change in Henle's fiber layer that was overlooked before. 


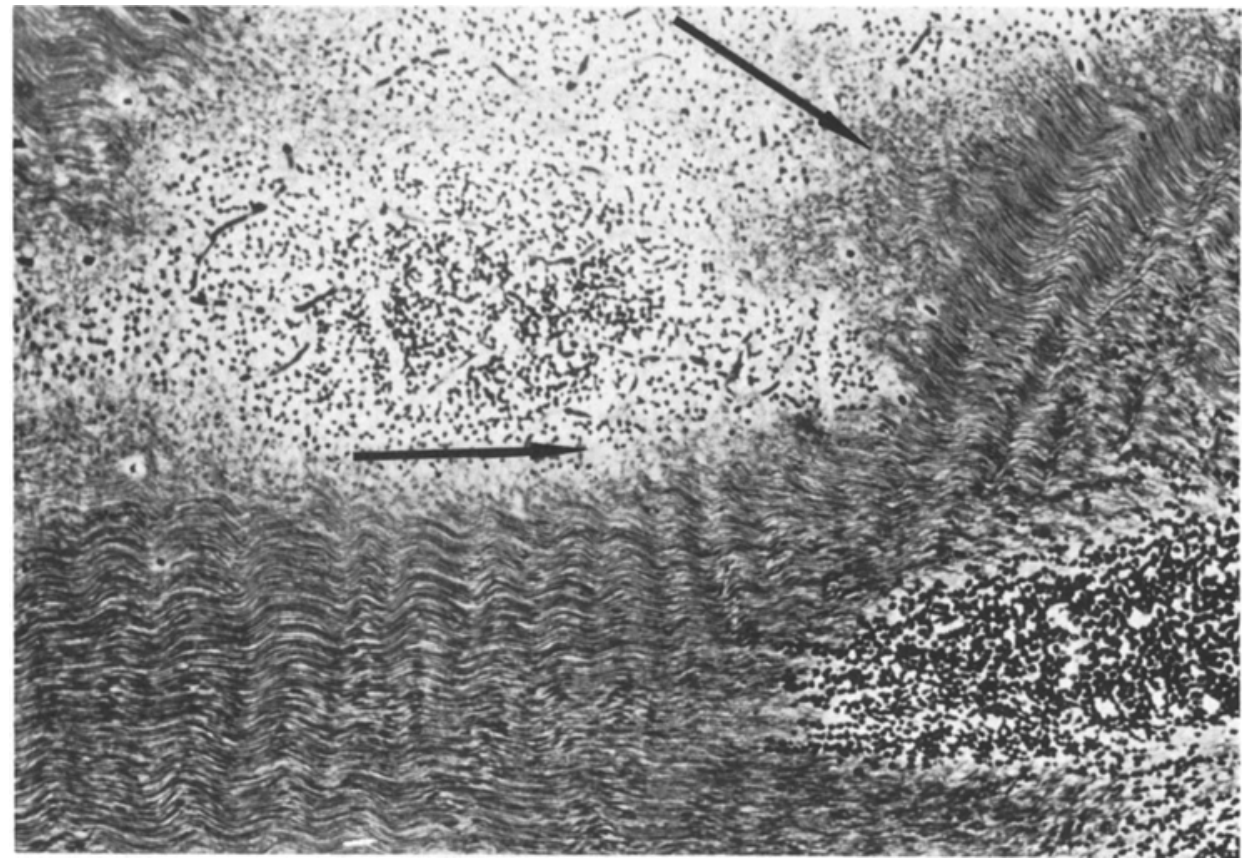

Fig. 6. (Wolter) The direction of the waves in Henle's fiber layer on the temporal side and on the upper inner aspect is indicated by arrows to show the concentric curving. Islands of inner nuclear layers are seen in the interspaces. - Frozen flat section, Hortega stain, photomicrograph $\times 150$

Flat sections in the zone of the temporal aspect of the fovea clearly show the horizontal folds in Bruch's membrane (Fig. 2). Parallel sections also reveal the folds in the inner retinal layer. However, in the foveal zone these folds of the inner retina run in a somewhat irregular vertical direction (Fig. 3). While the horizontal choroidal folds are rather regular and parallel to each other, the vertical folds of the inner retina in the foveal zone are somewhat irregular and at an angle to each other.

The surprising new observation in the old slides is that Henle's fiber layer in its temporal half and in the center exhibits a very regular wavy pattern (Fig. 4). This reminds of well combed wavy hair. In the temporal aspect of the fovea the direction of these small waves is at right angles to the horizontal folds of Bruch's membrane and parallel to the retinal folds of this zone. Careful study, however, shows that the direction of these waves is vertical only on the temporal aspect of the fovea (Fig. 5). The very regular waves follow the contour of the round foveola in concentric lines (Figs. 4, 5, 6 and 7). In one of the frozen sections this is difficult to see, but Henle's fiber layer can be reconstructed in several of these flat sections and this reconstruction confirms the fact that the foveola is surrounded by very regular concentric rings of "microwaves" involving Henle's fibers. The very center appears flat and without distortion. This center, of course, is represented by the cell bodies of the foveolar cones.

\section{Discussion}

Tumor pressure from behind the eye in the present case has caused extensive horizontal folding of Bruch's membrane. These horizontal choroidal folds extend 


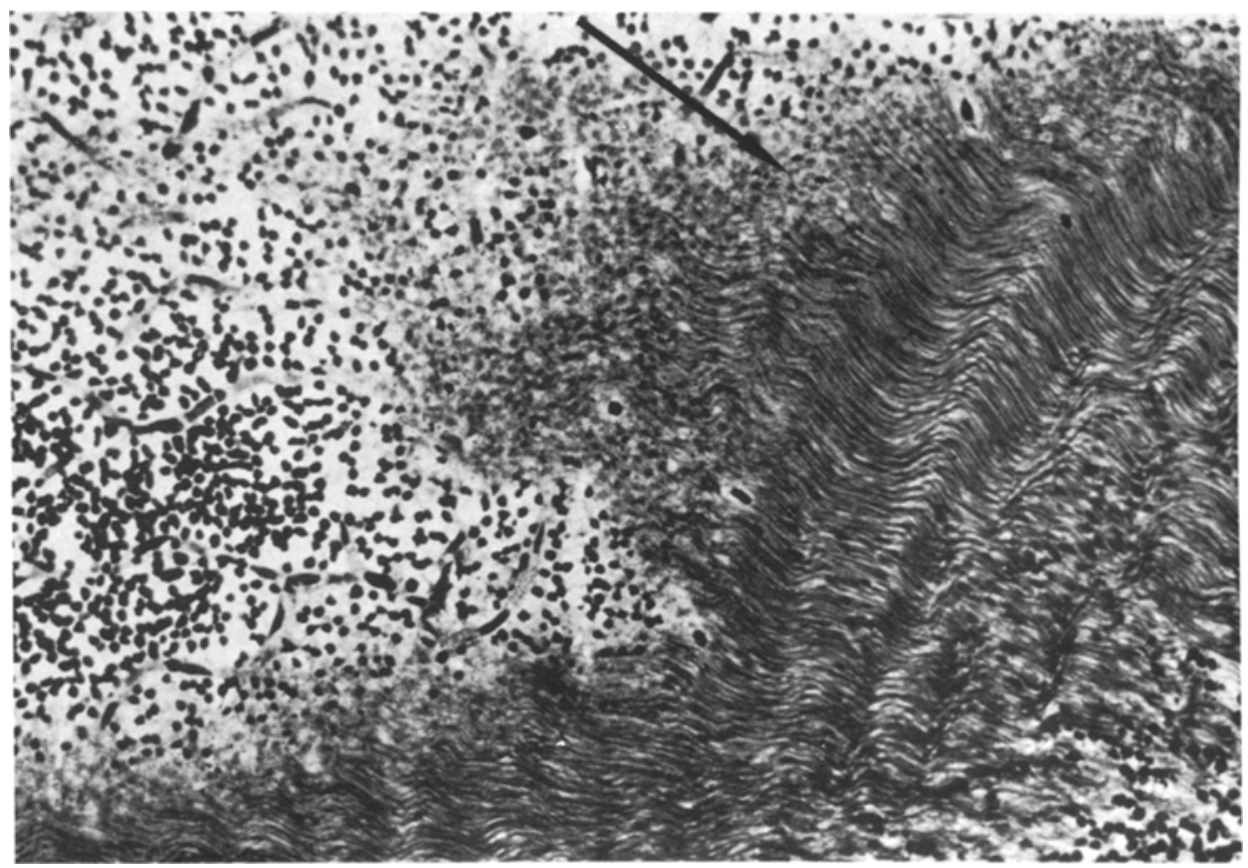

Fig. 7. (Wolter) High power view of the concentrically curving lines of waves in Henle's layer at high power. Inner nuclear layer with capillaries containing blood on the left. - Flat frozen section, Hortega stain, photomicrograph $\times 300$

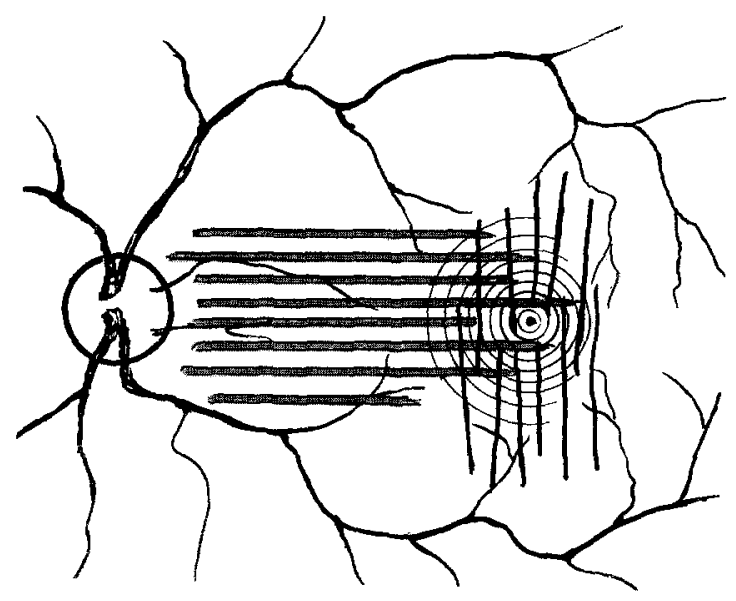

Fig. 8. (Wolter) Drawing to demonstrate the relationship of the horizontal folds of the inner choroid and pigment epithelium, the vertical folds of the retina in the foveal region and the overlapping concentric waves in Henle's fiber layer 
into the temporal half of the fovea (Fig. 8). This fact was seen clinically and confirmed by routine histologic study. In the region between disk and fovea the inner retinal layers are also found to be folded horizontally and parallel to the choroidal folds. In the region of the fovea, however, the folds of the inner retina change in direction to a somewhat irregular vertical arrangement. Henle's fiber layer, finally, does not take part in the horizontal or in the vertical folding. It exhibits a circular and very regular folding pattern of its own, that looks like wavy hair or corrugated metal. The concentric waves in Henle's fiber layer are much smaller - have a higher "frequency" - than the horizontal or vertical folds. Thus, I call them "microwaves". These concentric microwaves are clearly centered on the foveola. Henle's fibers seen in a flat section of a normal eye are perfectly straight and resemble the rays of a star-burst, of course. It is easy to imagine the change in the present case resulting from pressure on the posterior eye if one would assume Henle's fibers to be non-elastic. The much more interesting question is whether or not this waving of Henle's fibers indicates a protective function of Henle's fibers against foveolar distortion. The foveola is normally suspended in Henle's fibers, of course. Henle's fibers may act like a breakwater between the horizontal folds of the choroid and the foveal vertical folds of the retina and transform these coarse wrinkles of opposite directions into small and orderly microwaves which preserve normal shape and order in the foveola. A great ability for mechanical adjustment in general is one of the outstanding capabilities of Henle's fiber layer. In early central retinal edema, for example, it is Henle's fiber layer that "stands up" and, thus, makes space for much fluid accumulation without great disturbance of central vision [14]. The deposition of exudate and lipids in Henle's fiber layer in the formation of the so-called star figure is another example for the adjustability of this structure [15].

The reported change is a new observation in old slides. The situation of the case and the lucky sections will be difficult to repeat. However, new studies of Henle's fiber layer possibly functioning as a mechanical buffer to protect the foveola may be indicated.

\section{References}

1. Dellaporta A (1955) Fundus changes in hypotony. Amer J Ophth 40:781-785

2. Gass JDM (1970) Stereoscopic Atlas of Macular Diseases. C.V. Mosby, St Louis, p 98

3. Hedges TJ, Leopold IH (1959) Parallel retinal folds. Their significance in orbital spacetaking lesions. Arch Ophth 62:353-355

4. Kroll AJ, Norton EWD (1970) Regression of choroidal folds. Trans Amer Acad Ophth Otolaryngol 74:515-526

5. Newell FW (1973) Choroidal folds. Amer J Ophth 75:930-942

6. Norton EWD (1969) A characteristic fluorescein angiographic pattern in choroidal folds. Proc R Soc Med 62:119-128

7. Pannu JS, Lawson LJ (1973) Acute transient chorioretinal folds. Ann Ophth 5:880-882

8. Schepens CL, Schwartz A (1958) Intraocular tumors. Arch Ophth 60:72-83

9. Vedel-Jensen N (1969) Retinal grooves caused by pressure on the globe. Acta Ophth 59:37-39

10. Wolter JR, Jampel RS (1957) Faltenbildung der Netzhaut durch Tumordruck auf den Bulbus, Klin Monatsbl Augenbeilk 131:433-438 
11. Wolter JR (1962) Parallel horizontal retinal folding. Amer J Ophth 53:26-29

12. Wolter JR, Bryson JM, Blackhurst RT (1966) Primary orbital melanoma. The Eye Ear Nose and Throat Monthly 45:64-67

13. Wolter JR (1974) Parallel Horizontal Choroidal Folds Secondary to an Orbital Tumor. Amer J Ophth 77:669-673

14. Wolter JR: Cystoid macular edema: a study of histopathological details. Ophthalmic Surgery in print

15. Wolter JR, Goldsmith RI, Phillips RL (1957): Histopathology of the star figure of the macular area in diabetic and angiospastic retinopathy. Arch Ophth 57:376-385

Received September 23, 1980 